\title{
Mechanism of Oxidative Decarboxylation of Substituted Mandelic Acids by Alkaline Sodium Hypochlorite
}

\author{
Robert Eliason, ${ }^{\mathrm{a}, \star}$ Jeffery Platz ${ }^{\mathrm{a}}$ and Per H. J. Carlsen ${ }^{\mathrm{b}}$ \\ a Southwest State University, Chemistry Department, Marshall, Minnesota 56258, USA and ' University of Trondheim, The Norwegian \\ Institute of Technology, Institute of Organic Chemistry, N-7034 Trondheim, Norway
}

\begin{abstract}
Eliason, R., Platz, J. and Carlsen, P. H. J., 1991. Mechanism of Oxidative Decarboxylation of Substituted Mandelic Acids by Alkaline Sodium Hypochlorite. - Acta Chem. Scand. 45: 491-493.

The mechanism of oxidative decarboxylation of 2-hydroxy-2-phenylethanoic acid (mandelic acid) by aqueous alkaline sodium hypochlorite (commercial bleach) has been studied. Kinetic studies for a series of 4-substituted mandelic acids $\left(\mathrm{CH}_{3} \mathrm{O}, \mathrm{CH}_{3}\right.$, $\mathrm{H}, \mathrm{F}, \mathrm{Cl}, \mathrm{CF}_{3}, \mathrm{NO}_{2}$ substituents) gave a Hammett $\varrho$ value of -0.23 . When deuterium was substituted for hydrogen on the $\alpha$-carbon atom of mandelic acid, a secondary kinetic isotope effect, $\left(k_{\mathrm{H}} / k_{\mathrm{D}}\right)$, of 1.07 was obtained. On the basis of these results a model for the transition state is proposed.
\end{abstract}

The oxidative decarboxylation of 2-hydroxycarboxylic acids by acidic or basic aqueous solutions of hypohalite ion has been used synthetically to produce aldehydes or ketones. ${ }^{1.2}$ Pink and Stewart ${ }^{3}$ studied the mechanism of this reaction using alkaline bromine water. They proposed a mechanism in which the carboxylate anion attacked either $\mathrm{Br}_{2}$ or $\mathrm{HOBr}$ to form the $\mathrm{R}_{2} \mathrm{C}(\mathrm{OH}) \mathrm{COOBr}$ intermediate in a pre-equilibrium step. The products were then produced from rate-determining abstraction of the hydroxy hydrogen by a base $\left(\mathrm{OH}^{-}, \mathrm{Br}^{-}\right.$or $\left.\mathrm{H}_{2} \mathrm{O}\right)$. They also concluded that $\mathrm{Br}_{2}$ was a much more effective oxidizing agent than $\mathrm{HOBr}$ and that $\mathrm{OBr}^{-}$was virtually inert. The same reaction has been studied by Gupta and Grover ${ }^{4}$ using aqueous chlorine. The effect of $\mathrm{pH}$ on the reaction was examined, and it was found that the reaction had a maximum rate at a $\mathrm{pH}$ of 4.4 and at a $\mathrm{pH}$ of 8.3 . They proposed a mechanism in which the carboxylate ion was attacked by either hypochlorous acid or molecular chlorine in a rate-determining step to produce the products. They obtained a value of $28 \mathrm{~J} \mathrm{~mol}^{-1}$ $\mathrm{K}^{-1}$ for the entropy of activation which they interpreted as indicating that the reaction occurred between oppositely charged ions. A mechanism involving free radicals was ruled out. We undertook a study of the oxidative decarboxylation of 2-hydroxy-2-phenylethanoic acid (hereafter referred to as mandelic acid) with alkaline aqueous sodium hypochlorite in order better to describe the nature of the rate determining step of the reaction.

\section{Results and discussion}

Assuming that the mechanism suggested by Pink and Stew$\mathrm{art}^{3}$ is applicable to oxidative decarboxylation by alkaline sodium hypochlorite, the stepwise mechanism for mandelic acid would be given by eqns. (1)-(5) where $S$ represents the

\footnotetext{
* To whom correspondence should be addressed.
}

$$
\begin{aligned}
& \mathrm{S}^{-}+\mathrm{HOCl} \underset{k_{-1}}{\stackrel{k_{1}}{\rightleftarrows}} \mathrm{SCl}+\mathrm{OH}^{-} \\
& \mathrm{S}^{-}+\mathrm{Cl}_{2} \underset{k_{-2}}{\stackrel{k_{2}}{\rightleftarrows}} \mathrm{SCl}+\mathrm{Cl}^{-} \\
& \mathrm{SCl}+\mathrm{H}_{2} \mathrm{O} \stackrel{k_{3}}{\longrightarrow} \mathrm{S}^{-}+\mathrm{HOCl}+\mathrm{H}^{+} \\
& \mathrm{SCl}+\mathrm{OH}^{-} \stackrel{k_{4}}{\longrightarrow} \text { Products } \\
& \mathrm{SCl}+\mathrm{OCl}^{-} \stackrel{k_{5}}{\longrightarrow} \text { Products }
\end{aligned}
$$

mandelate group. Since the previous investigators ${ }^{3.4}$ determined that the kinetics of the oxidation reaction was first order in mandelic acid and first order in oxidant, we decided to simplify the kinetic experiments by running the reactions under pseudo-first-order conditions ${ }^{5}$ by keeping the hypochlorite concentration at least 50 times greater than the mandelate concentration. In this case the rate law would be given by $k_{\text {obs }}$ [mandelate]. In order to verify the order with respect to mandelate ion, kinetic experiments were run in triplicate at mandelate concentrations of $7.54 \times 10^{-3}$ and $3.77 \times 10^{-3} \mathrm{M}$. The $k_{\text {obs }}$ values of $4.7(4)$ $\times 10^{-5}$ and $5.6(1) \times 10^{-5} \mathrm{~s}^{-1}$, respectively, were obtained. The reactions were followed for at least four half-lives, and the subsequent plots of $\ln$ [mandelate] vs. time were linear over this period. While the agreement of the rate constants is slightly outside the experimental error, it was assumed that the reaction was first order in mandelic acid.

Oxidative decarboxylation by chlorine or bromine water has been shown to be very sensitive to $\mathrm{pH} .{ }^{3.4}$ Consequently, all kinetic measurements were made at the same $\mathrm{pH}$. The commercial bleach that was used as the source of sodium 


\section{ELIASON ET AL.}

hypochlorite had a pH of 12.44. A stock solution for each of the substituted mandelic acids was prepared using standardized aqueous sodium hydroxide as the solvent. The $\mathrm{pH}$ for these solutions was adjusted to $12.4(1)$. The $\mathrm{pH}$ of three typical kinetic reactions were monitored, and it was observed that under these conditions the $\mathrm{pH}$ of the reaction mixture remained constant throughout the reaction.

In the Pink and Stewart mechanism ${ }^{3}$ the rate-determining step involves attack by either hydroxide or hypochlorite ion on $\mathrm{SCl}$, eqns. (4) and (5). However, at the $\mathrm{pH}$ of the experiments the concentration of both chlorine and hypochlorous acid would be expected to be very low. It could, thus, be argued that the formation of $\mathrm{SCl}$ from the reaction of chlorine and hypochlorous acid with mandelate ion, eqns. (1) and (2), might be rate determining. The concentrations of these species estimated from the hydrolysis con$\operatorname{stant}^{6}\left(3.94 \times 10^{-4} \mathrm{M}^{2}\right)$ for chlorine and the dissociation constant $^{7}\left(2.95 \times 10^{-8} \mathrm{M}\right)$ for hypochlorous acid were calculated to be about $3.5 \times 10^{-5} \mathrm{M}$ for $\left[\mathrm{Cl}_{2}\right]$ and about $6.9 \times 10^{-6} \mathrm{M}$ for $[\mathrm{HClO}]$. The extremely small chlorine concentration would seem to preclude any significant reaction via that route. If the reaction given in eqn. (1) is rate determining, then the rate $=k_{2}$ [mandelate] $[\mathrm{HClO}]$, and $k_{2}$ $=k_{\mathrm{obs}} /[\mathrm{HClO}]$ where $k_{\mathrm{obs}}$ is the observed pseudo-first-order rate constant. The calculated second-order rate constant $\left(k_{2}\right)$ in this case would be about $8.5 \mathrm{M}^{-1} \mathrm{~s}^{-1}$. This value is of the same magnitude of that determined $\left(k_{2}=5.5 \mathrm{M}^{-1} \mathrm{~s}^{-1}\right)$ by Gupta and Grover ${ }^{4}$ under conditions using an excess of mandelate ion.

A Hammett plot of $k_{\mathrm{obs}}^{\prime} / k_{\mathrm{obs}}^{\circ}$ vs. $\sigma$, where $k_{\mathrm{obs}}^{\prime}$ is the pseudo-first-order rate constant for the substituted compound and $k_{\mathrm{obs}}^{\circ}$ is the analogous constant for the non-substituted compound, gave a least-squares slope, $\mathrm{Q}$, of $-0.23(4)$, Fig. 1. The small $\varrho$ value indicates that a change in substituent has little effect on the reaction rate. Any of the possibilities for the rate-determining step are supported by this observation. If eqn. (1) or (2) represented the rate-determining step, it would be expected that $\varrho$ would be

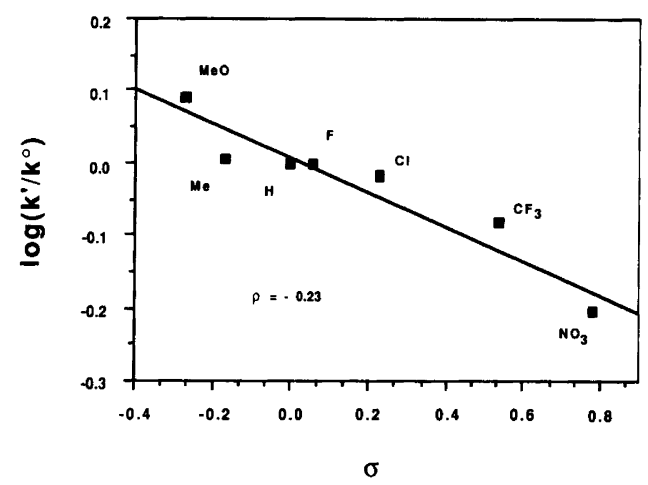

Fig. 1. A Hammett plot for the reaction of $p$-substituted mandelic acids with alkaline sodium hypochlorite. The rate constants $k^{\prime}$ and $k^{\circ}$ represent the substituted and hydrogen-substituted mandelic acids, respectively.

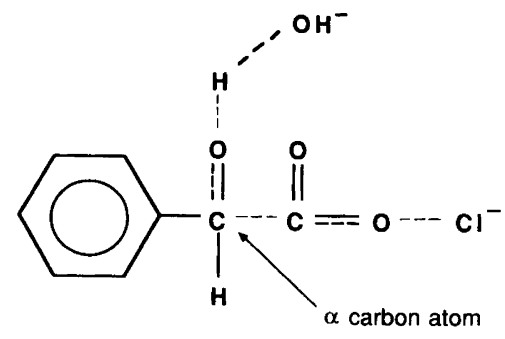

Fig. 2. Model of transition state of the reaction of mandelic acid with alkaline sodium hypochlorite.

about the same magnitude as the $\varrho$ value for ionization of mandelic acid but opposite in sign. Electron-withdrawing substituents would stabilize the mandelate anion while electron donation would destabilize it. Relative to each other the more stable compound would react more slowly than the less stable compound. The $\varrho$ value for mandelic acid ionization is not known, but the value for phenylacetic acids is $0.5 .^{8}$ Thus, it would be predicted that the substituent effect would give a small negative $\varrho$ value. If eqns. (4) and (5) were rate determining as proposed by Pink and Stewart, ${ }^{3}$ a possible model for the transition state would be bond reorganization to produce an aldehyde carbonyl. If the transition state lay toward the product side, then this partially formed carbonyl carbon would contain a partially positive charge due to bond polarization by the oxygen atom. Electron-donating substitutents would stabilize this forming carbonyl which would result in a lower activation energy and an increase in rate. An electron-withdrawing substituent would have the opposite effect. The expected $\varrho$ value would be negative and small in magnitude.

A kinetic isotope effect was used further to serve as a probe of the transition state. If either eqns. (1) or (2) represented the rate-determining step of this reaction, substitution of a deuterium atom for hydrogen at the $\alpha$-carbon atom in mandelic acid would yield no kinetic isotope effect. However, if eqns. (4) or (5) are rate determining, then $\alpha$-deuterium isotope effect would be predicted. The $\alpha$ carbon atom undergoes a change in hybridization from $\mathrm{sp}^{3}$ to $\mathrm{sp}^{2}$ during the course of the reaction. The isotope effect for going from an $\mathrm{sp}^{3}$ hybridized carbon to an $\mathrm{sp}^{2}$ hybridized carbon has been calculated theoretically to be about 1.4 at room temperature. ${ }^{9 a}$ In practice, experimental values this large are usually not found for this type of isotope effect. When the hydrogen is attached to a secondary carbon, experimental values for $\alpha$-deuterium isotope effects, $\left(k_{\mathrm{H}} / k_{\mathrm{D}}\right)_{\text {obs }}$, range from 1.09 to $1.2 .^{9 \mathrm{~b}}$ In the present case the experimental isotope effect, $\left(k_{\mathrm{H}} / k_{\mathrm{D}}\right)_{\text {obs }}$, was determined to be 1.075(72) uncorrected for the amount of residual hydrogen in the deuteriated mandelic acid. Correction for the $8 \% \mathrm{H}$ in the $\left[{ }^{2} \mathrm{H}\right]$ mandelic acid gives an isotope effect of 1.083. While this isotope effect is slightly smaller than might be expected, it still supports the conclusion that the transition state must involve substantial carbonyl character ( $\mathrm{sp}^{2}$ hybridization) at the $\alpha$-carbon atom. 
Taking the value for $\varrho$ and the isotope effect together suggests that eqns. (4) and (5) represent the rate-determining steps. A model for the transition state of these ratedetermining steps is shown in Fig. 2. Simultaneous with proton abstraction of the hydroxy hydrogen by a base $\left(\mathrm{OH}^{-}\right.$or $\mathrm{ClO}^{-}$in this case) there is substantial bond reorganization in which a carbon-carbon bond and oxygenchlorine bond are broken and in which two carbon-oxygen double bonds are formed.

\section{Experimental}

General. ${ }^{1} \mathrm{H}$ NMR spectra were recorded on a JEOL FX-100 NMR spectrometer. IR spectra were obtained from a Perkin-Elmer 257 grating spectrometer or a Nicolet SXC-20 FTIR spectrometer. Mass spectra were recorded on an AEI MS-902 spectrometer at $70 \mathrm{eV}$. GLC analyses were performed on a Varian 3700 gas chromatograph equipped with a BP-1 capillary column (length, $25 \mathrm{~m}$ ) or on a packed $5 \% \mathrm{OV}-17$ column (length, $1.6 \mathrm{~m}$ ).

4-Substituted mandelic acids were prepared by hydrolysis of the corresponding mandelonitriles obtained from the appropriate $p$-substituted benzaldehydes according to the procedure described by Carrara, et al. ${ }^{10}$

[ $\alpha{ }^{2} H$ ]Mandelic acid. A mixture containing $3.04 \mathrm{~g}$ of mandelic acid and $3.20 \mathrm{~g}$ of dry barium oxide in $20 \mathrm{ml}$ of $\mathrm{D}_{2} \mathrm{O}$ $(99.8 \% \mathrm{D})$ was refluxed for $3 \mathrm{~h}$. The reaction mixture was then cooled to room temperature, added to $50 \mathrm{ml}$ of diethyl ether and with stirring acidified with conc. hydrochloric acid. The ether layer was then separated and the aqueous phase extracted three times with $20 \mathrm{ml}$ portions of ether. The combined ether layers were washed three times with 5 $\mathrm{ml}$ portions of water and then dried over anhydrous magnesium sulfate. The solution was filtered, and the solvent was evaporated under reduced pressure. The residue was recrystallized from toluene to yield $2.4 \mathrm{~g}$ ( $71 \%$ ) of the pure product, m.p. $121-122^{\circ} \mathrm{C}$. The isotopic content was determined by mass spectrometry to be $92 \% \mathrm{D}$.

${ }^{1} \mathrm{H}$ NMR (100 MHz, $\mathrm{CDCl}_{3}$ ): $\delta$ 7.5-7.3 (arom. multiplet). MS [IP $70 \mathrm{eV} \mathrm{(m/z, \%} \mathrm{rel.} \mathrm{int.)]:} 154$ (1.7), 153 (19), 152 (1.5), 108 (100), 107 (7). IR (KBr): 3399, 2938, $2679,1711,1333,1279,1217,1093,954,896,869,730,697$, $602,528 \mathrm{~cm}^{-1}$.

Oxidation of $\left[\alpha^{2} \mathrm{H}\right]$ mandelic acid with sodium hypochlorite. This reaction was performed under conditions similar to those used in the kinetics experiments. From $154 \mathrm{~g}$ of $\left[\alpha-{ }^{2} \mathrm{H}\right]$ mandelic acid and after work-up including molecular distillation were obtained $55 \mathrm{mg}$ of $\alpha$-deuteriobenzaldehyde with a D-content of $93 \%$. MS [IP $70 \mathrm{eV}(\mathrm{m} / \mathrm{z}, \%$ rel. int.)]: 108 (10.2), 107 (100), 106 (16), 105 (98). IR (neat): 3084, 3062, 3030, 2106, 2068, 2051, 1687, 1587, 1583, 1351, $1312,1231,1214,1166,1072,1021,999,939,828,791,733$, $688,641 \mathrm{~cm}^{-1}$.
Kinetic procedures. Commercial bleach (unstabilized) was purchased and standardized. The $\mathrm{pH}$ of the bleach was determined to be 12.4 . The amount of hypochlorite present was determined by titration with standardized sodium thiosulfate $^{11}$ to be $0.558 \mathrm{M}$. Stock solutions of the mandelic acids were prepared by weighing out the appropriate amount of the acid to give a $0.144 \mathrm{M}$ solution. Standardized sodium hydroxide was added to the acid and the mixture diluted with water to produce the appropriate concentration. This resulted in a stock solution of the substituted mandelate with a $\mathrm{pH}$ of 12.4 .

Kinetic solutions were prepared by mixing $5 \mathrm{ml}$ bleach and $75 \mathrm{ml}$ diethyl ether. The reactions were initiated by the addition of $2 \mathrm{ml}$ mandelate stock solution. The reaction was followed by removing an aliquot of ether periodically and measuring the production of aldehyde. The amount of aldehyde present in the ether was determined by measuring the absorption in the UV region at the appropriate wavelength of the aldehyde. The ether aliquot was returned to the reaction mixture after each measurement. All kinetic reactions were conducted in a constant temperature bath at $25.0(1)^{\circ} \mathrm{C}$.

The isotope experiments were carried out in pairs using the protium compound in one reaction and the deuterium analog in the other. The concentration of the mandelate substrates for both isomers was identical.

Benzaldehyde was identified as the sole reaction product when mandelic acid was oxidized on a preparative scale with sodium hypochlorite under the conditions used in the kinetic experiments.

\section{References}

1. Carlsen, P. H. J. Acta Chem. Scand., Ser. B 38 (1984) 343.

2. Nwaukwa, S. O. and Keehn, P. M. Tetrahedron Lett. 23 (1982) 3155.

3. Pink, J. M. and Stewart, R. Can. J. Chem. 49 (1971) 649.

4. Gupta, P. and Grover, K. C. J. Indian Chem. Soc. 50 (1973) 397.

5. Moore, J. W. and Pearson, R. G. Kinetics and Mechanism, Wiley, New York 1981, 3rd ed., p. 16.

6. Connick, R. E. and Chia, Y.-T. J. Am. Chem. Soc. 81 (1959) 1280.

7. Gordon, A. J. and Ford, R. A. The Chemist's Companion, Wiley, New York 1972, p. 59.

8. Jaffe, H. H. Chem. Rev. 53 (1953) 191.

9. Melander, L. and Saunders, W. H., Jr. Reaction Rates of Isotopic Molecules, Wiley, New York 1980 (a) p. 172, (b) p. 173.

10. Carrara, G., Fava, F., Martinuzzi, C. and Vecchi, A. Gazz. Chim. Ital. 82 (1952) 674.

11. Kolthoff, I. M. and Sandell, E. B. Textbook of Quantitative Inorganic Analysis, MacMillan, New York 1952, 3rd ed., p. 594.

Received August 13, 1990. 